\section{Aspectos diagnósticos da otite média com derrame na faixa etária pediátrica}

\section{Diagnostic aspects of otitis media with effusion in children}

Maria Helena Valente 1

Ana Maria de Ulhôa Escobar 2

Sandra Josefina Ferraz Ellero Grisi 3

\section{Abstract}

Objectives: to present and discuss diagnostic aspects of middle ear infection with effusion at a primary health care clinic.

Methods: research was carried out using the Medline, Lilacs and SciELO databases (1994-2004). Five hundred and twenty-three articles were found, of which 30 were considered for this review.

Results: the study revealed considerable disparity with regard to definitions, inconsistency in terms of clinical profile and a lack of standardization of diagnostic criteria. This has contributed to inadequate diagnosis of otitis media with effusion (OME). Evidence leading to a diagnosis of effusion were: a) at least two eardrum abnormalities relating to discoloration, darkening and mobility; and/or b) tympanic membrane (MT) typically retracted, concave, and with altered coloration; and/or c) reduced mobility or absence of mobility on pneumatic otoscopy (PO). The $P O$ was shown to be more accurate than simple otoscopy (SO), which is considered the method of choice for diagnosis of OME. When the pneumatic otoscopy is inconclusive, tympanometry may recommended, as this increases the accuracy of the diagnosis.

Conclusions: in clinical practice, diagnosis of OME should be carried out principally by way of pneumatic otoscopy, to which all children suspected of having OME should be submitted. Where there is doubt, tympanometry is recommended.

Key words Otitis media with effusion, Diagnosis, Pediatrics
1-3 Departamento de Pediatria. Faculdade de Medicina. Universidade de São Paulo. Rua Capote Valente, 432. Conj. 41. São Paulo, SP, Brasil. CEP: 05.409-001.

E-mail: valentemh@gmail.com

\section{Resumo}

Objetivos: apresentar e discutir aspectos diagnósticos da otite média com derrame (OMD) na prática clínica da atenção primária à saúde.

Métodos: foram pesquisadas as bases de dados Medline, Lilacs e SciELO (1994-2004). Identificados 523 artigos, dos quais 30 foram considerados para o presente trabalho.

Resultados: os estudos revelaram disparidade entre as definições, inconsistência no quadro clínico e falta de padronização dos critérios diagnósticos. Isto contribuiu para a inadequação diagnóstica da OMD. As evidências que levaram ao diagnóstico de efusão foram: a) pelo menos duas anormalidades do tímpano relacionadas à coloração, opacificação e mobilidade; elou b) membrana timpânica (MT) tipicamente retraída, côncava, e com alteração da coloração; elou c) mobilidade reduzida ou ausente na otoscopia pneumática $(O P)$. A OP mostrou ser mais acurada que a otoscopia simples (OS), sendo considerada como método de escolha para o diagnóstico clínico da OMD. Quando houver dúvida na otoscopia pneumática, pode-se indicar a timpanometria, que aumenta a acurácia deste diagnóstico.

Conclusões: na prática clínica, o diagnóstico da OMD deve ser realizado, primariamente pela otoscopia pneumática, em toda criança com suspeita de OMD. Em caso de dúvida deve-se indicar a timpanometria.

Palavras-chave Otite média com derrame, Diagnóstico, Pediatria 


\section{Introdução}

A otite média com derrame (OMD) é condição frequente na população pediátrica, e se caracteriza pela presença de fluído seroso ou mucoso no espaço da caixa do tímpano, sem sinais e sintomas de inflamação aguda na orelha média, que, geralmente, se estabelece após quadro diagnosticado ou não de otite média aguda (OMA).1,2 Pode ser observada em pelo menos uma ocasião em $91,1 \%$ das crianças antes dos dois anos de idade, $66 \%$ entre dois e cinco anos, e $22 \%$ entre cinco e 12 anos de idade, com a maioria dos episódios se resolvendo espontaneamente, sem qualquer tratamento, dentro de três meses. ${ }^{1-4}$ Porém, cerca de 30 a $40 \%$ das crianças sofrem episódios recorrentes de otite média com derrame, das quais 5 a $10 \%$ vivenciam episódios que duram um ano ou mais. ${ }^{1-4}$

Essa condição também é comum na prática cotidiana de clínicos como os pediatras e os médicos de saúde da família, os profissionais que mais precoce e frequentemente são visitados pelos pais para o atendimento das crianças nos primeiros anos de vida, seja para as supervisões da saúde ou para as eventuais intercorrências. $4-6$

A doença inflamatória na orelha média é um dos motivos mais comuns para as crianças pequenas visitarem o clínico e serem submetidas à cirurgia.1,4-6 Nos menores de três anos de idade, se estabelece como a causa mais comum de hipoacusia condutiva adquirida, com potencial efeito negativo sobre a aquisição da fala, linguagem e rendimento escolar, pelo que a precisão diagnóstica, assim como a individuação das crianças de risco para a efusão crônica na orelha média se tornam importantes. $1,2,4,7$

Embora seja um dos problemas clínicos de maior prevalência na prática clínica ambulatorial, o diagnóstico acurado da OMD não é simples e nem fácil, sendo permeado por dificuldades e incertezas, mesmo para profissionais experientes. 5,6

$\mathrm{Na}$ atualidade, a imprecisão diagnóstica da OMD é considerada como a pedra angular que contribui para as taxas alarmantes de resistência bacteriana aos antimicrobianos. 1-3,6 Diagnósticos incorretos, pela não diferenciação entre os quadros de OMD e OMA, contribuem para a prescrição indevida e excessiva de antibióticos, complicações da OMD e seguimento inadequado das crianças sob risco para o desenvolvimento de distúrbios da audição, fala e linguagem. ${ }^{1-4,7}$

Apesar das proposições das diretrizes diagnósticas, 35\% dos médicos desconsideram a presença de efusão na orelha média como critério relevante e prioritário para o diagnóstico dos quadros de otite média, resultando na prescrição abusiva de antibióticos, por profissionais com apenas $50 \%$ de certeza diagnóstica. $5,6,8,9$

Ainda que existam orientações específicas para identificar o tratamento, não se observa unanimidade sobre como realizar o diagnóstico da OMD.5,6,9 Evidentemente, devem ser discutidos a realização de estudos científicos, consensos e diretrizes sobre a OMD, sobre a qual se observa variabilidade nas definições, inconsistência do quadro clínico, falta de padronização dos critérios diagnósticos, desconhecimento da acurácia dos métodos e a discutível proficiência do examinador, que, conjuntamente, contribuem para os diagnósticos incorretos. 1,4,6-8

O objetivo desta revisão é o de discutir aspectos importantes relacionados à acurácia diagnóstica da otite média com derrame na infância, na prática clínica da atenção primária à saúde.

A relevância justifica-se pela alta prevalência da OMD na infância, diagnosticada por profissionais que não são especialistas em otorrinolaringologia, como os médicos da saúde da família e pediatras. 1,4 $\mathrm{Na}$ prática clínica diária é de fundamental importância diferenciar a otite média com derrame da otite média aguda, uma vez que a confusão entre os quadros resulta na prescrição indevida e abusiva de antibióticos, aumento da resistência bacteriana e nas consequências negativas do seguimento inadequado dos quadros crônicos da OMD.1,5,6

\section{Métodos}

Foram pesquisadas as bases de dados Medline da National Library of Medicine (EUA), através do Pubmed, Lilacs (Literatura Latino-Americana e do Caribe em Ciências da Saúde) e SciELO (Scientific Eletronic Library Online) utilizando-se os descritores "otite média com derrame", "otite média secretora", "otite média secretória", "otite média serosa", "derrame do ouvido médio", "diagnóstico" e "pediatria", e os termos correspondentes em inglês, "otitis media with effusion", "diagnosis", "pediatrics", na faixa etária pediátrica de zero a dezoito anos de idade, de janeiro de 1994 a dezembro de 2004, nos idiomas inglês, espanhol e português.

A busca inicial identificou no Medline do Pubmed 481, no Lilacs 40 e no SciELO 2 referências de artigos publicados relacionando a "otite média com derrame", "diagnóstico" e "pediatria", no período referido.

Os critérios de inclusão analisaram estudos randomizados, coorte, caso-controle, observacionais descritivos ou analíticos que apresentaram aspectos diagnósticos da OMD na atenção primária à saúde. 
Os critérios de exclusão consideraram as investigações sobre o tratamento, complicações, aspectos subjetivos do quadro clínico da OMD, métodos diagnósticos pouco usuais na prática clínica, tais como as otoemissões acústicas, timpanocentese e a timpanostomia, por serem da competência do especialista, e indicados em situações específicas. Além dessas, foram excluídas as referências que não possuíam abstracts; os relatos de casos clínicos e as cartas ao editor.

Os autores elegeram variáveis relacionadas à definição, critérios de avaliação e diagnóstico, acurácia dos principais métodos diagnósticos e a questão da habilitação médica, por consi-derar que a discussão sobre tais aspectos poderia se relacionar ao uso consciente, explícito e criterioso de aspectos envolvidos com as decisões clínicas do médico da atenção primária no diagnóstico da OMD, o que significaria a coerência do cuidado com o paciente. A realização da revisão envolveu o trabalho de dois pesquisadores, que avaliaram, de forma independente, a qualidade metodológica de cada artigo selecionado, através da elaboração de um protocolo de pesquisa que incluiu itens sobre como os estudos foram encontrados, variáveis referentes aos pacientes envolvidos nos estudos, como a idade, variável sobre as condições do diagnóstico como indivíduo da comunidade, escola, ambulatório ou em situação pré-cirúrgica, critérios de inclusão e exclusão dos artigos, definição dos desfechos de interesse, verificação da acurácia dos resultados, determinação da qualidade dos estudos, e análise estatística utilizada.

Na busca, 523 abstracts satisfizeram os critérios de inclusão. Desses, foram excluídos 66 por envolveram doenças específicas que evoluem com OMD, 220 por abordarem o tratamento e as complicações e 98 por tratarem dos aspectos subjetivos do quadro clínico. Os autores optaram pela exclusão de 109 estudos referentes aos métodos diagnósticos pouco utilizados na prática clínica cotidiana, disponíveis apenas para o especialista ou em situações de pesquisa.

Completou-se a revisão através de minuciosa análise da bibliografia contida nos artigos obtidos pelo método acima, sendo selecionadas pelo abstract 30 investigações relacionadas diretamente às questões diagnósticas de otite média com derrame na atenção primária à saúde.

\section{Resultados}

O diagnóstico da OMD foi realizado por vários métodos: quadro clínico, otoscopia simples e pneumática, timpanometria clássica e quantitativa, minitimpanometria, reflexometria acústica, audiometria, otoemissões acústicas, vídeo-otoscopia, otomicroscopia, microtimpanoscopia binocular, timpanocentese e miringotomia, isoladamente ou em combinação uns com os outros. 1,3,4,10-12

$\mathrm{Na}$ prática clínica da atenção primária à saúde, porém, o diagnóstico da OMD foi realizado pelo quadro clínico e exame de otoscopia simples.1,10-12 No entanto, esses métodos se mostraram inconsistentes e inadequados, uma vez que os trabalhos revisados evidenciaram variabilidade das definições, inconsistência do quadro clínico, diversidade dos critérios de avaliação e de diagnóstico, controversa acurácia das principais ferramentas diagnósticas, e da proficiência do examinador que, acrescidas da ansiedade parental, não cooperação infantil e equipamentos comprometidos, contribuíram para a inadequação do diagnóstico.1-3,8,9,10-12

As variações sutis das definições encontradas para a OMD1,10-12 foram expostas na Tabela 1.

A OMD foi considerada, geralmente, como oligo ou assintomática.10-16 Condições inespecíficas das vias aéreas superiores, como obstrução nasal persistente, rinofaringites virais frequentes, respiração bucal, rinite alérgica e as otites médias de repetição foram associadas ao quadro de OMD. 8,13 Crianças com OMD apresentaram cinco vezes mais episódios de otite média aguda do que aquelas sem efusão na orelha média.2,8 Hipoacusia, plenitude ou "estalido" nas orelhas, sensação de ouvido "tapado", irritabilidade, otalgia discreta intermitente, alterações do equilíbrio e motricidade, e distúrbios do sono, atenção, linguagem e aprendizado também foram observados nas crianças maiores. 1,4,7,8,14-17

A inexpressividade do quadro clínico e a inexistência de um "padrão-ouro", prontamente disponível nos vários componentes do exame físico de rotina para o diagnóstico da doença da orelha média com derrame, orientou como procedimento padrão para o diagnóstico primário na prática clínica os recursos semiológicos da otoscopia simples (OS) e/ou pneumática (OP), ${ }^{18,19}$ com a avaliação indireta da membrana timpânica e caixa do tímpano. $1,3,4,10-$ 12,18,19 Assim, foi recomendado para o clínico da atenção primária realizar a otoscopia sempre que possível, em todas as crianças doentes assim como nas sadias, durante as supervisões de saúde.1,1012,18,19 E os médicos deveriam estar aptos e treinados a realizar a otoscopia pneumática que é a ferramenta mais apropriada para o diagnóstico da OMD na atenção primária à saúde.5,6,9

$\mathrm{Na} \mathrm{OMD}$, inicialmente se detecta a efusão na orelha média através da membrana timpânica (MT), 
Tabela 1

Definições da otite média com derrame (OMD).

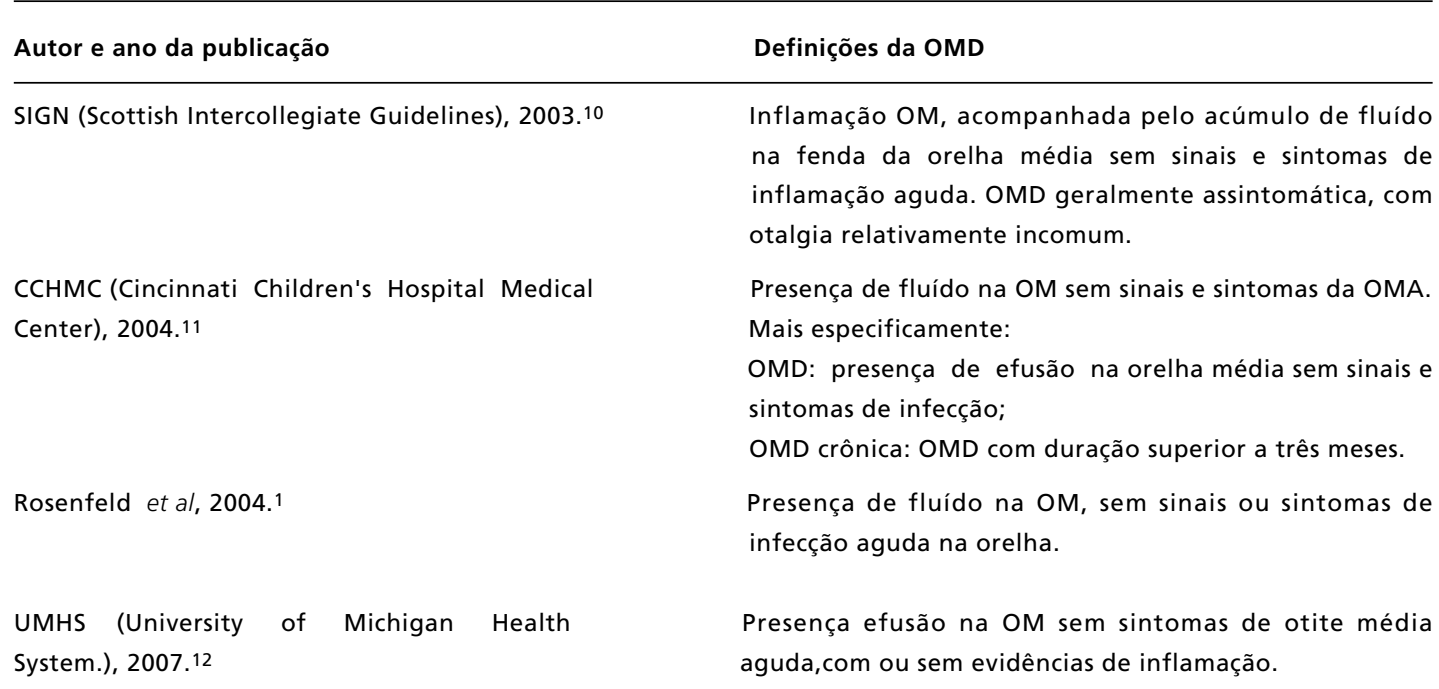

OM=orelha média; $\mathrm{OMD=otite} \mathrm{média} \mathrm{com} \mathrm{derrame;} \mathrm{OMA=otite} \mathrm{média} \mathrm{aguda.}$

segundo o conjunto de alterações inflamatórias específicas relacionadas à cor, aspecto, textura do tímpano, presença de exsudado, e eventualmente bolhas na caixa do tímpano. $1,4,5,10-12,18,19$

Os aspectos importantes da otoscopia simples e pneumática $20-28$ a serem considerados pelo clínico para o diagnóstico da OMD foram descritos na Tabela 2 .

$\mathrm{Na}$ otoscopia, a presença de qualquer uma das três condições descritas abaixo indicou a presença da OMD: $1,10-12,18-28$

a) Pelo menos duas alterações da MT relacionadas com a: coloração anormal, descrita como amarela, âmbar, ou azul; opacificação não devida à cicatriz; e diminuição ou ausência da mobilidade da MT, 1, 10-12,18-28 e/ou;

b) Aspectos típicos da OMD como a MT côncava/retraída, reflexo luminoso alterado e mudança de cor para tipicamente amarela ou âmbar.1,4,8,,10-12,18-21 Com iluminação adequada, pode-se visualizar a presença de bolhas de ar ou nível ar/líquido.1,10-12,18-21,29 Plenitude e abaulamento, embora não característicos, podem ser encontrados 10-12,18-21, e/ou;

c) Pneumo-otoscopia com a mobilidade da MT reduzida ou ausente.1,10-12,18,19,22-28

A horizontalização da proeminência do processo curto ou lateral do maléolo foi considerada como sinal objetivo e útil na detecção da OMD, pela pressão negativa na orelha média, que "repuxa" medialmente o processo longo do maléolo. 19
A acurácia de cada uma das características do tímpano na detecção da efusão na orelha média mostrou valores de sensibilidade e especificidade para a hiperemia/opacificação de 64 e 36\%, abaulamento/retração de 56 e $93 \%$, nível ar-fluido de 12 a $100 \%$ e para a mobilidade prejudicada de 75 a 90\%. 18,19,22-26 A maioria dos sinais observados na membrana timpânica através da OP mostrou valor limitado na detecção de efusão na orelha média, com a mobilidade do tímpano diminuída à valores moderados de sensibilidade.22-27

Em relação ao tipo de otoscopia, quando a OP é realizada por indivíduos qualificados com equipamento adequado, e os resultados comparados com aqueles da miringotomia, a combinação dos diferentes sinais evidenciou valores de sensibilidade e especificidade aceitáveis, de $81 \%$ e $86 \%$ na determinação da presença de efusão na orelha média,22-26 enquanto a OS, sem testar a mobilidade do tímpano, mostrou valores de 60 e $70 \%$, respectivamente. 20,21 Por isso, estudos de coorte recomendam, enfaticamente, a utilização da OP pelo clínico nos diagnósticos na atenção primária à saúde, como método diagnóstico principal da OMD. 10-12,22-28

O diagnóstico da OMD nos menores de seis meses de idade pela OS, mesmo se realizado por avaliadores experientes, mostrou ser pouco acurado. ${ }^{23}$ A otomicroscopia no ambulatório de pediatria revelou acurácia igual ou maior a $90 \%$, se realizada pelo otorrinolaringologista. 28

A timpanometria, como medida da imitâncio 
A otoscopia no diagnóstico da otite média com derrame.

\begin{tabular}{|c|c|c|c|c|}
\hline $\begin{array}{l}\text { Autor e ano da } \\
\text { publicação }\end{array}$ & $\begin{array}{l}\text { Desfecho estudado } \\
\text { (OMD) }\end{array}$ & $\begin{array}{l}\text { População } \\
\text { (crianças) }\end{array}$ & Metodologia & Tipos de dados obtidos \\
\hline Franche et al., 1998.20 & Acurácia OS & 98 & OS e timpanometria & $\begin{array}{l}\text { OS: } S=87,5 \% \text { e } \\
E=61 \%\end{array}$ \\
\hline Engel et al., 2000.21 & Acurácia OS & 250 & OS e timpanometria & $\begin{array}{l}\text { OS: 0-6 meses } \\
\text { idade: índice } \\
\text { Карpa<0,4 }\end{array}$ \\
\hline de Melker et al., 1993.22 & Acurácia OP & 111 & OP e timpanometria & $\begin{array}{l}\text { OP: MT "imóvel" } \\
\text { S=83\% } \\
\text { "Pouca mobilidade" } \\
\text { S= } 97 \%\end{array}$ \\
\hline Alho et al., 1998.23 & $\begin{array}{c}\text { Acurácia OP e } \\
\text { minitimpanometria }\end{array}$ & 354 & OP e minitimpanometria & $\begin{array}{l}\text { OP: Mobilidade } \\
\text { "ausente": } S=81 \% \text {, } \\
E=86 \%\end{array}$ \\
\hline Guo et al., 2002.24 & $\begin{array}{c}\text { Acurácia OP, RA, } \\
\text { timpanometria e } \\
\text { videotoscopia }\end{array}$ & 89 & $\begin{array}{l}\text { OP, timpanometria, } \\
\text { RA, videotoscopia }\end{array}$ & OP: $S=95 \%$ e $E=85 \%$ \\
\hline Nozza et al., 1994.25 & Acurácia OP & 171 & OP e timpanometria & OP: $S=86 \%$ e $E=71 \%$ \\
\hline Koivunen et al., 1999.26 & $\begin{array}{c}\text { Acurácia OP e } \\
\text { aspectos MT }\end{array}$ & 76 & OP e timpanometria & OP: $S=81 \%$ e $E=86 \%$ \\
\hline Field et al., 1993.27 & $\begin{array}{c}\text { Acurácia } \\
\text { otomicroscopia, } \\
\text { minitimpanometria, } \\
\text { e timpanometria } \\
\text { clássica }\end{array}$ & 50 & $\begin{array}{l}\text { Otomicroscopia, } \\
\text { minitimpanometria } \\
\text { e timpanometria } \\
\text { clássica }\end{array}$ & $\begin{array}{l}\text { Otomicroscopia: } \\
\mathrm{S}=90 \% \text { e } E=92 \%\end{array}$ \\
\hline Isaac et al., 1999.28 & $\begin{array}{l}\text { Acurácia } \\
\text { otomicroscopia } \\
\text { e timpanometria }\end{array}$ & 36 & $\begin{array}{l}\text { Otomicroscopia, } \\
\text { timpanometria, RA }\end{array}$ & $\begin{array}{l}\text { Otomicroscopia: } \\
\text { S=100\% e E=98\% }\end{array}$ \\
\hline
\end{tabular}

OMD=otite média com derrame; $\mathrm{OP}=$ otoscopia pneumática; $O S=0$ toscopia simples; $R A=$ reflexometria acústica; $\mathrm{MT}=$ membrana timpânica; $\mathrm{S}=$ sensibilidade; $\mathrm{E}=$ especificidade. 
acústica do canal auditivo externo e da orelha média, avaliou de forma objetiva o estado da orelha média.24,25,30-36 Através do comportamento do tímpano, submetido às pressões positivas e negativas, descrito por gráficos denominados de timpanogramas, a doença da orelha média aumentou a rigidez do tímpano. $27,30-37$

A utilização pelo clínico do exame de timpanometria ou imitânciometria acústica foi amplamente incentivada como método objetivo, adjunto e/ou complementar à otoscopia, a ser indicado sempre que persistir qualquer dúvida sobre o estado da orelha média da criança. ${ }^{5,6,9,30-37}$ Assim, o diagnóstico definitivo de OMD na atenção primária à saúde infantil foi definido por estes dois procedimentos; a otoscopia e a timpanometria.20-28,30-37

Aspectos práticos de interesse para o clínico relacionados à timpanometria ou imitânciometria acústica23-25,27,30-37 na faixa etária pediátrica foram apresentados na Tabela 3.

A classificação mais utilizada na prática clínica considerou como anormal o timpanograma com curva $\mathrm{B}$, que sugere a presença de efusão na caixa do tímpano, e a curva A como normal, indicando a orelha média aerada. Alem desses, a curva tipo $\mathrm{C}$, subdividida em $\mathrm{C}_{1}$ e $\mathrm{C}_{2}$, significou pressão negativa na orelha média, com o ponto de corte em -300 decapascais $(\mathrm{daPa})$, com alguns considerando a presença de efusão na orelha média no tipo $\mathrm{C}_{2} \cdot 27,34,37$

A timpanometria de mesa ou clássica só pode ser realizada por profissional especializado como o fonoaudiólogo.23-25,30-32,35-37 A minitimpanometria, ferramenta portátil desenvolvida para ser utilizada na atenção primária, se mostrou dependente da cooperação infantil.27,33 Esses métodos apresentaram sensibilidade e especificidade de $70-91 \%$ e $63-98 \%$, respectivamente. $23-25,27,30-34$

Além de avaliar o estado da orelha média, o timpanograma do tipo B também sugere perda auditiva condutiva maior do que 25 decibéis $(\mathrm{dB})$, sendo empregada como ferramenta de triagem populacional, seja para avaliar o estado da orelha média, seja como teste inicial de triagem auditiva populacional, permitindo a identificação de $95 \%$ das perdas auditivas infantis, além de reduzir $69 \%$ da carga de audiometria. 37

A reflexometria acústica (RA), assim como a timpanometria, também foi considerada como método diagnóstico objetivo, adjunto e/ou complementar da otoscopia, onde a presença de bolhas e nível líquido na caixa do tímpano, características da OMD, diminuíram a acurácia do método.24,38,39

Os critérios de avaliação e diagnóstico da OMD utilizados pelos diferentes grupos de pesquisa também apresentaram pequenas variações, com diferenças na escolha dos métodos e na ênfase dada a cada um deles, refletindo os objetivos específicos de cada investigação. Os principais modelos de avaliação e diagnóstico, 1,10-12,40-46 com suas prioridades, concordâncias e divergências foram registrados na Tabela 4.

À heterogeneidade das definições, objetivos e critérios de avaliação e diagnósticos somaram-se os diferentes contextos de recrutamento da criança, com quadros de complexidades diversas, e diagnósticos realizados por profissionais com diferentes habilitações. ${ }^{8,10-12,24,40-46}$

Nos últimos anos, com as inúmeras diretrizes clínicas enfatizando a importância da otoscopia no diagnóstico da OMD,1,10-12 a literatura passou a discutir a qualidade do diagnóstico da doença da orelha média, enfatizando os pré-requisitos necessários para o diagnóstico acurado.40-46 Questões relacionadas com a habilidade e competência do profissional 9,40-46 para o diagnóstico, capacitação e treinamentos em otoscopia, proficiência no diagnóstico, critérios utilizados, obediência às diretrizes propostas, comparação entre a acurácia da OP e OS, utilização prática das ferramentas diagnósticas começaram a ser discutidas, com a perspectiva do clínico diferenciar entre a orelha média normal daquelas com efusão, e entre os quadros de OMD e OMA, com alguns desses aspectos contemplados na Tabela 5 .

Estudos de casos e controles definiram como risco para a OMD as seguintes crianças: as que frequentam creche; as que têm irmãos mais velhos com otites de repetição; as filhas de pais fumantes e aquelas com problemas de audição e comportamento. $2 \mathrm{O}$ conjunto das informações constatou que em 40 a $50 \%$ dos casos, nem as crianças afetadas e nem seus pais referiram queixas significativas relacionadas com a perda auditiva de intensidade leve a moderada secundária ao quadro de OMD.1,17

Observa-se, portanto, que os autores consideram que o médico da atenção primária à saúde deve estar treinado para realizar a otoscopia simples e pneumática, com a consideração de que a avaliação do prejuízo do movimento da MT aumenta a acurácia do diagnóstico da otite média com derrame na faixa etária pediátrica. ${ }^{40-46} \mathrm{Em}$ caso de dúvida diagnóstica, orienta-se solicitar exames complementares como timpanometria e/ou reflexometria acústica.24,25,27,30-37 Reitera-se que o diagnóstico correto da OMD diminui o uso indevido de antimicrobianos e previne sequelas importantes na faixa etária pediátrica como hipoacusia e distúrbios de linguagem. 10-12,40-46 
Tabela 3

A timpanometria no diagnóstico da otite média com derrame.

\begin{tabular}{|c|c|c|c|c|}
\hline $\begin{array}{l}\text { Autor e ano da } \\
\text { publicação }\end{array}$ & $\begin{array}{l}\text { Desfecho } \\
\text { estudado }\end{array}$ & $\begin{array}{r}\text { População } \\
\text { (crianças) }\end{array}$ & Metodologia & Tipos de dados obtidos \\
\hline Alho et al., 1998.23 & Tipo B & 354 & $\begin{array}{c}\text { Timpanometria e } \\
\text { otoscopia }\end{array}$ & $S=79 \%$ e $E=93 \%$ \\
\hline Guo, Shiao, 2002.24 & Tipo B & 89 & $\begin{array}{l}\text { Timpanometria e } \\
\text { otoscopia }\end{array}$ & $\mathrm{S}=91 \%$ e $E=85 \%$ \\
\hline Nozza et al., 1994.25 & Extensão > 275 & 171 & $\begin{array}{l}\text { Timpanometria } \\
\text { quantitativa }\end{array}$ & $S=81 \%$ e $E=82 \%$ \\
\hline Field et al., 1993.27 & Tipo $B+C_{2}$ & 50 & $\begin{array}{l}\text { Minitimpanometria e } \\
\text { timpanometria clássica }\end{array}$ & $\begin{array}{c}\text { Timpanometria mesa: } B+C_{2} \text { : } \\
\text { S=94.4\% e } E=71.8 \% \\
\text { Microtimpanometria: } B+C \\
S=100 \% \text { e } E=75 \% \\
\text { Apenas } B: S=83.4 \% \text { e } E=75 \%\end{array}$ \\
\hline Sassen et al., 1994.30 & Tipo B & $\begin{array}{l}41 \text { ( }<2 \text { anos) e } \\
221 \text { ( }>2 \text { anos) }\end{array}$ & Timpanometria & $\begin{array}{c}\text { Crianças <2 anos: } \mathrm{S}=90 \% \text { e } \\
\mathrm{E}=67 \% \\
\text { Crianças }>2 \text { anos: } \mathrm{S}=81 \% \text { e } \\
\mathrm{E}=63 \%\end{array}$ \\
\hline Palmu et al., 1999. 31 & Tipo B & 58 & $\begin{array}{l}\text { Timpanometria } \\
\text { e otoscopia }\end{array}$ & $S=70 \%$ e $E=98 \%$ \\
\hline Watters et al.; 1997.32 & Tipo B & 501 & Timpanometria & $S=91 \%$ e $E=79 \%$ \\
\hline Koivunen et al., 1997.33 & Tipo B & $\begin{array}{r}206 \text { (PA) e } 162 \\
\text { (pré-cirúrgicas) }\end{array}$ & $\begin{array}{l}\text { Minitimpanometria e } \\
\text { clássica }\end{array}$ & $\begin{array}{c}\text { Cooperativas: } \mathrm{S}=79 \% \text { e } \\
\text { E }=93 \% \\
\text { Não cooperativas: } S=71 \% \text { e } \\
\text { E }=38 \%\end{array}$ \\
\hline van Balen et al., 1994.34 & Tipo B e $C_{2}$ & 142 & $\begin{array}{l}\text { Minitimpanometria e } \\
\text { clássica }\end{array}$ & $S=94 \%$ e $E=48 \%$ \\
\hline Palmu et al., 2002.35 & $\begin{array}{l}\text { Admitâncio, } \\
\text { pico e extensão }\end{array}$ & 2.497 & $\begin{array}{l}\text { Timpanometrias } \\
\text { seriadas }\end{array}$ & $\begin{array}{l}\text { OM e TV: aumentam } \\
\text { admitâncio e diminuem } \\
\text { extensão }\end{array}$ \\
\hline Palmu et al., 2001. 36 & Pico $<100 \mathrm{daPa}$ & 329 & $\begin{array}{l}\text { Timpanometrias } \\
\text { seriadas }\end{array}$ & $\begin{array}{c}\text { Pico <100da: marcador para } \\
\text { OM }\end{array}$ \\
\hline MRC, 1999.37 & Tipos $B+B$ e $B+C_{2}$ & 1153 & $\begin{array}{l}\text { B bilateral, } \mathrm{B}+\mathrm{C}_{2} \mathrm{e} \\
\text { audiometria }\end{array}$ & $\begin{array}{c}\mathrm{B}+\mathrm{B}: \text { diagnóstico } 90 \% \\
\text { hipoacusias } \\
\mathrm{B}+\mathrm{C}_{2}: \text { diagnóstico } 95 \% \\
\text { hipoacusias }\end{array}$ \\
\hline
\end{tabular}

daPa=decapascal; E=especificidade; $M R C=$ Multi-Centre Otitis Media Study Group (Grupo no Reino Unido constituído por 16 departamentos $\mathrm{ORL}$ ); OM=otite média; $\mathrm{OMD}=$ otite média com derrame; $\mathrm{ORL}=$ otorrinolaringologista; $\mathrm{PA}=$ pronto atendimento; $S=$ sensibilidade; Tipo $A=$ timpanograma normal, indicando orelha média aerada; Tipo $B=$ timpanograma anormal, indicando orelha média efundida; Tipo $C=$ timpanograma que indica pressão negativa, com a orelha média efundida ou não; Tipo $C_{2}=$ timpanograma com grande pressão negativa, e grande probabilidade da presença da efusão na orelha; $\mathrm{TV}=$ tubo de ventilação. 
Tabela 4

Critérios de avaliação e diagnóstico da otite média com derrame (OMD).

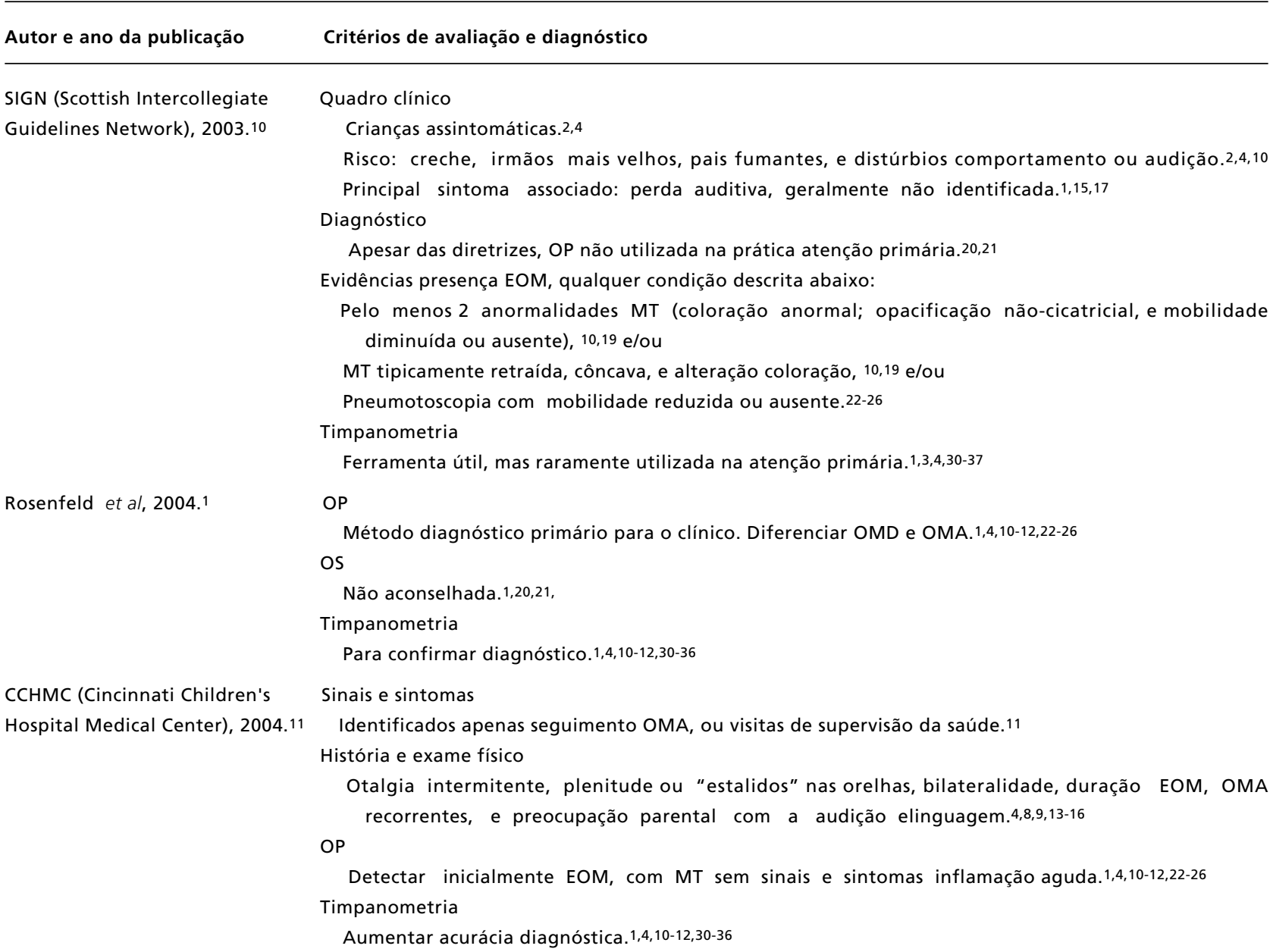

UMHS (University of Michigan Health System.), 2007.12
Fundamentos das recomendações diagnósticas

Distinguir entre OMA e OMD. OMA exige sintomas: dor ou febre com inflamação EOM.1,2,4 Diagnóstico da presença EOM

Combinar OS, OP, timpanometria até não haver dúvida EOM.1,4,10-12,22-26

Diagnóstico da OMD

MT toda criança saudável ou doente. 12

OP ou timpanometria sempre que possível.1,4,10-12,22-26

Quando existe EOM

Determinar natureza EOM para distinguir derrames transitórios da persistentes. 12

Técnicas identificação OMD

Questão central é otoscopia definir se EOM ou não, na ausência sintomas agudos.1,4,10-12,22-26

Presença ou ausência EOM incerta, utilizar todas técnicas: OS, OP, e timpano metria.20-26,30-36 OP

Ferramenta técnica fundamental diagnóstico OMA e OME.1,4,10-12,22-26

Clínico habilitado em otoscopia

OP com S e E=70 a $90 \%$, e a OS com 60 a $70 \%$ para EOM, respectivamente. $10-12,40-46$

Timpanometria

Valioso método adjunto, mas não substituto da OS e OP. Confirmação objetiva EOM.1,24,25,27,30-37

Útil para CG aperfeiçoar habilidade otoscopia.30-37

S e E de 70 a $90 \%$ para EOM nas crianças cooperativas.30-37

Curva A e otoscopia normal predizem adequadamente ausência EOM.30-37

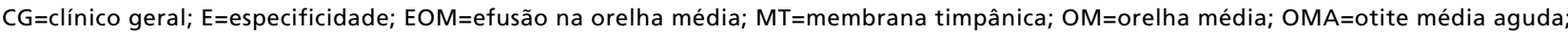

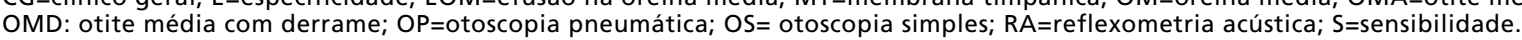


Tabela 5

Questões relacionadas à qualidade do diagnóstico da otite média com derrame.

\begin{tabular}{|c|c|c|c|c|}
\hline $\begin{array}{l}\text { Autor e ano da } \\
\text { publicação }\end{array}$ & $\begin{array}{l}\text { Desfecho } \\
\text { estudado }\end{array}$ & $\begin{array}{l}\text { População } \\
\text { (médicos) }\end{array}$ & Metodologia & Tipos de dados obtidos \\
\hline $\begin{array}{l}\text { Blomgren, } \\
\text { Pitkäranta, } 2003.9\end{array}$ & $\begin{array}{l}\text { Avaliar } \\
\text { concordância CG } \\
\text { e residente ORL }\end{array}$ & $\begin{array}{l}50 \text { avaliações } \\
\text { de CG e ORL }\end{array}$ & $\begin{array}{l}\text { ORL fotos } 50 \mathrm{MT} \text {, } \\
\text { e } 2 \text { clínicos } \\
\text { avaliaram fotos, } \\
\text { OP e }\end{array}$ & $\begin{array}{l}\text { Diagnóstico OMA: ORL }=44 \% \text { e } C G=64 \% \\
\text { Para o diagnóstico: } C G \text { viu sintomas e cor MT e ORL: } \\
\text { movimento e posição M pela OP } \\
\text { Timpanometria reduziu } 30 \% \text { OMA }\end{array}$ \\
\hline $\begin{array}{l}\text { Silva, Hotaling, } \\
1997.40\end{array}$ & Proficiência OP & $\begin{array}{l}4 \text { residentes, } \\
\text { ORL }\end{array}$ & $\begin{array}{l}\text { OP, } \\
\text { otomicroscopia, } \\
\text { miringotomia. }\end{array}$ & $\begin{array}{l}\text { Treinamento e proficiência na OP: } S>80 \% \text { e } E>70 \% \\
\text { Em otomicroscopia: } S>90 \% \text { e } E>80 \%\end{array}$ \\
\hline $\begin{array}{l}\text { Jones, Kaleida, } \\
2003.41\end{array}$ & $\begin{array}{l}\text { Comparar } \\
\text { acurácia OP } \\
\text { com OS }\end{array}$ & $\begin{array}{l}34 \text { médicos } \\
\text { residentes }\end{array}$ & $\begin{array}{l}50 \text { vídeos OS e } \\
\text { diagnóstico. } \mathrm{Na} \\
\text { sequência, OP e } \\
\text { diagnóstico. }\end{array}$ & $\begin{array}{l}\text { Acurácia OP }>\text { OS. Melhora absoluta da OS }(61 \%) \\
\text { para OP }(76 \%)=15 \% \\
\text { Melhora relativa da OS para OP }=26 \% \\
\text { Melhora relativa da } S \text { e da E da OS para OP }=24 \% \\
\text { e } 42 \% \text {, respectivamente }\end{array}$ \\
\hline $\begin{array}{l}\text { Pichichero, } \\
\text { Poole, 2001.42 }\end{array}$ & $\begin{array}{l}\text { Distinguir OMA } \\
\text { e MT normal, } \\
\text { OMA e a OMD. }\end{array}$ & $\begin{array}{l}514 \text { pediatras } \\
\text { e ORL }\end{array}$ & $\begin{array}{l}9 \text { vídeos para DD } \\
\text { entre OMA, OMD, } \\
\text { MT normal }\end{array}$ & $\begin{array}{l}\text { Diagnóstico correto: pediatra } 50 \% \text { e ORL } 73 \% \text {. } \\
\text { Pediatra e ORL: ausência normal em } 89 \text { a } 100 \% \text {, e } 93 \\
\text { a } 100 \% \text { tempo, respectivamente }\end{array}$ \\
\hline $\begin{array}{l}\text { Pichichero, } \\
2002.43\end{array}$ & $\begin{array}{l}\text { Acurácia DD, } \\
\text { timpanocentese } \\
\text { e atb }\end{array}$ & $\begin{array}{l}383 \text { médicos } \\
\text { residentes } \\
\text { pediatria USA }\end{array}$ & $\begin{array}{l}9 \text { vídeos, } \\
\text { timpanocentese e } \\
\text { atb e }\end{array}$ & $\begin{array}{l}\text { Diagnóstico correto: } 41 \% \pm 16 \% \text { Timpanocentese: } \\
89 \% \text {, Atb apropriado: } 78 \%\end{array}$ \\
\hline $\begin{array}{l}\text { Pichichero, } \\
2003.44\end{array}$ & $\begin{array}{l}\text { Diferenciar } \\
\text { OMA, OMD com } \\
\text { OP }\end{array}$ & $\begin{array}{l}2407 \text { médicos } \\
\text { pediatras }\end{array}$ & Vídeo e OP & $\begin{array}{l}\text { Pediatras superdiagnosticaram OMA, e confundiram } \\
\text { OMA e OME }\end{array}$ \\
\hline $\begin{array}{l}\text { Steinbach et al., } \\
2002.45\end{array}$ & $\begin{array}{l}\text { Habilidades } \\
\text { residentes } \\
\text { pediatria } \\
\text { diagnóstico }\end{array}$ & $\begin{array}{l}27 \text { médicos } \\
\text { residentes } \\
\text { pediatria e } \\
2 \text { ORL }\end{array}$ & $\begin{array}{l}\text { Avaliação clínica } \\
\text { OP e } \\
\text { timpanometria }\end{array}$ & $\begin{array}{l}\text { Residente pediatria e ORL: Kappa grupal }=0,30 \\
\text { Maior correlação individual: hiperemia com Kappa } \\
=0,40 \\
\text { Pior correlação: posição MT, Kappa }=0,16\end{array}$ \\
\hline $\begin{array}{l}\text { MacClements et al, } \\
2002.46\end{array}$ & $\begin{array}{l}\text { Ferramentas } \\
\text { diagnósticas na } \\
\text { prática }\end{array}$ & $\begin{array}{l}316 \text { médicos } \\
\text { atenção primária }\end{array}$ & Questionários & $\begin{array}{l}\text { Prática: OP }=66 \% \text {, timpanometria }=29 \% \\
\text { OP: } 55 \% \text { residentes medicina de família não reali- } \\
\text { zam } \\
\text { Falta treinamento e equipamento. }\end{array}$ \\
\hline
\end{tabular}

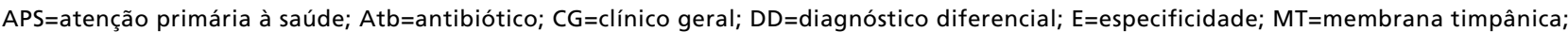
$\mathrm{OM}=$ otite média; $\mathrm{OMA}=$ otite média aguda; $\mathrm{OMD=otite} \mathrm{média} \mathrm{com} \mathrm{derrame;} \mathrm{OP=otoscopia} \mathrm{pneumática;} \mathrm{OS=otoscopia} \mathrm{simples;}$ ORL=otorrinolaringologia; $S=$ sensibilidade. 


\section{Discussão}

$\mathrm{O}$ atendimento à população pediátrica se constitui num importante desafio no contexto da atenção primária à saúde, com a doença da orelha média se estabelecendo como um dos principais diagnósticos na população infantil menor de três anos de idade.1,2,4-6 A alta prevalência da doença da orelha média e suas implicações fizeram com que a Organização Mundial da Saúde considerasse as habilidades relacionadas ao diagnóstico e tratamento da otite média na infância como uma prioridade a ser desenvolvida pelos profissionais da atenção primária à saúde. 47

A situação diagnóstica até o final dos anos 1990 podia ser definida como caótica. Até então, o objetivo principal era o tratamento da doença da orelha média, trazendo o "complexo otite média" como objeto de estudo, com a OMD e a OMA avaliadas conjuntamente, sem haver preocupação com o diagnóstico e a diferenciação entre os quadros de otite média.1-4 $\mathrm{Na}$ era da resistência antimicrobiana, a prescrição inadequada de antibióticos para os quadros de efusão crônica na orelha média e as repercussões deletérias na elevação da resistência aos antimicrobianos no mundo trouxeram como prioridade a identificação das crianças com OMD. 1,5,6,10-12,40-46 Assim, a importância da acurácia diagnóstica passou a ser valorizada, reacendendo a discussão sobre os critérios utilizados para diagnosticar e diferenciar os quadros de otite média entre si.5,6,40-46

Somente a partir do ano 2000, estudos baseados nas evidências disponíveis passaram a ser mais específicos e dirigidos no sentido de considerar a necessidade de debater aspectos envolvidos com a melhora da acurácia diagnóstica da OMD na criança e diferenciação dos quadros de otite média entre si, contribuindo para a competência dos clínicos que atuam na atenção primária à saúde, como o pediatra e o médico do Programa de Saúde da Família, os profissionais que mais precoce e intensamente têm contato com as crianças e suas famílias nos primeiros anos de vida. 1,4,6,40-46

O número de publicações relacionadas com a OMD continua aumentando significativamente nos últimos dois anos, apontando dificuldades relacionadas ao diagnóstico e tratamento, permeadas pela ausência de uma definição uniforme e homogênea do quadro nos grupos de pacientes incluídos nos estudos clínicos. Mesmo com a maioria dos autores considerando a presença de secreção na caixa do tímpano ou na fenda da orelha média para o diagnóstico da OMD, referida como um "acúmulo" ou "presença de fluído", ou "efusão", observou-se variabilidade na descrição da natureza do processo de base da efusão, descrita ora como "inflamação aguda", ora como "infecção aguda", evidenciando a existência de inconsistência na terminologia usada para se descrever o quadro. 1,3,4-6,912,44-48 Provavelmente, tal polêmica seja reflexo da discussão sobre a fisiopatologia do quadro, que anteriormente considerou a efusão como estéril, e em relação a qual, atualmente, se reconhece a presença de bactérias, complexamente organizadas, dentro de biofilmes, com comunicações intercelulares, predispondo à resistência aos antibióticos, ao mesmo tempo em que induzem à reação inflamatória, com papel importante na persistência da efusão da OMD e suas complicações. 47 Ampliando a possibilidade de confusão, alguns referiram o quadro de OMD como "sem sinais ou sem evidências otite média aguda", como se o diagnóstico do quadro agudo estivesse estabelecido e fosse consenso entre os vários autores. 1,4,10-12,40-46 A referência "sem evidência de processo inflamatório" traz a dúvida de como seria realizado o diagnóstico.1,4,10-12,47 Ao considerar o tempo de efusão na orelha média maior de três meses para definir o quadro de OMD crônica, buscam-se limitar a administração indevida de antibióticos para aqueles quadros com efusão transitória, assim como ressaltar a necessidade de vigilância e seguimento adequados da efusão crônica.1,4,9-17

O quadro clínico da OMD se mostrou diferente daquele observado na OMA, 1,4,9-17 apresentando-se, geralmente, oligo ou assintomático. $\mathrm{Na}$ prática clínica, cursou de forma silenciosa, com limites pouco nítidos, mutável, com a falta de sinais e sintomas se estabelecendo como um obstáculo ao diagnóstico.1,4,7,9-17 A observação de sinais e sintomas somente foi possível a partir do seguimento sistematizado dos quadros de OMA.1,2,4,8,10-12 O diagnóstico clínico, geralmente, foi realizado nas visitas de supervisão de saúde da criança, através de eventuais achados anormais na otoscopia. 1,2,4,9,18,19

Do ponto de vista prático, tornou-se mandatório para o clínico afastar o quadro nas crianças em situação de risco, assim como naquelas com episódios de OMA de repetição, rinite alérgica, comportamentos de desequilíbrio, insegurança nas situações que exigem maior coordenação motora, "desajeitadas e estabanadas" e, com alta frequência de acidentes para afastar a OMD.1,2,4,10-16 O fato do diagnóstico da OMD passar a maximizar a importância do desenvolvimento da linguagem infantil e a relativizar os danos estruturais da orelha média, solicita aos clínicos da atenção primária à saúde competências teóricas para o exercício da 
vigilância sobre o desenvolvimento normal da audição, fala e linguagem infantis, nos primeiros anos de vida. $1,4,7,8,14-16$

A limitada sensibilidade dos pais e cuidadores na detecção da perda auditiva leve e moderada deve desencorajar tanto os pesquisadores como os clínicos a utilizarem essa ferramenta para o diagnóstico e seguimento da OMD. 17 No entanto, a literatura insiste na valorização de qualquer preocupação parental relacionada com a audição, fala e linguagem infantil, que deve ser considerada e investigada, assim como a percepção médica de eventuais atrasos e/ou distúrbios nessas áreas, que podem ser consequentes à perda auditiva secundária à OMD. 1,4,7,14-17

$\mathrm{O}$ quadro clínico incipiente repassou à otoscopia a importância capital no diagnóstico da OMD, sendo aconselhado para que todo médico avalie a MT e orelha média, sempre que possível, em qualquer nível de atenção, tanto da criança doente como daquela saudável, que comparece à supervisão de saúde, independentemente de queixas e observações. 10-12,18,19

A necessidade da avaliação do estado da orelha média para o diagnóstico da OMD trouxe para o clínico uma questão técnica fundamental, que se refere, inicialmente, à questão de afastar ou afirmar a presença da efusão na orelha média.1,5,6,9,10-12,20-29 Para o cumprimento dessa determinação inicial imprescindível, o clínico não deve hesitar em empregar todas as técnicas disponíveis, como a otoscopia simples e/ou a pneumática e a timpanometria. 1,4,10-12,20-27,30-37

A otoscopia pneumática foi apontada como a ferramenta mais acurada na detecção da efusão na orelha média.22-27 Desta forma, tornou-se a principal ferramenta diagnóstica do clínico da atenção primária. ${ }^{22-26} \mathrm{O}$ prejuízo do movimento associado às alterações observadas na MT indicou a OP como o método essencial e mais acurado para o diagnóstico da OMD. 1,10-12,18,19,22-26 No entanto, no nosso meio, apesar das recomendações exaustivas, a maioria dos clínicos utiliza a otoscopia simples para os seus diagnósticos, mesmo considerando que vários autores desaconselham a utilização isolada desse procedimento para o diagnóstico do estado da orelha média.20,21 A desconsideração da mobilidade do tímpano para o diagnóstico da OMD, com a valorização imprópria somente da cor e do aspecto da membrana contribuiu para diagnósticos incorretos, uma vez que se detectou efusão na orelha média em apenas metade das membranas opacificadas, espessadas e de aspecto crônico.18,19,42-46 Além disso, a valorização da hiperemia inespecífica do tímpano, com valor preditivo pobre no diagnós- tico da OMA, foi observada em 5\% das OMD.9,44-46

Atualmente se orienta para o clínico, após a detecção da efusão na orelha média, tentar diagnosticar o tipo de efusão na caixa do tímpano, distinguindo entre os derrames transitórios, como o seroso ou purulento, daquelas efusões mucóides crônicas persistentes, associadas com morbidade significativa.22,23,26 As crianças com efusões transitórias devem ser reavaliadas em três meses, em conjunto com a observação criteriosa da sua linguagem, enquanto aquelas com derrames mucóides, geralmente associadas a danos anatômicos, como a otite adesiva ou bolsas de retração, devem ser referidas ao especialista. 18,22,23,26

As orientações e os critérios diagnósticos, apesar de semelhantes, diferem na priorização e ênfase dadas a cada um dos métodos.1,10-16,40-48 Alguns ainda chegaram a considerar o quadro clínico como ferramenta diagnóstica, enquanto outros indicaram a otoscopia como método primário de diagnóstico da OMD, praticamente desconsiderando o quadro clínico. 1,8,9,10-12,22-26 Todos os estudos foram unânimes em ressaltar a importância da detecção inicial da efusão na orelha média pela otoscopia, enfatizando a opção pela OP em relação a OS, justificada pela maior acurácia diagnóstica da pneumootoscopia. 1,8,9-12,18,19,20-26

Em relação às orientações e critérios diagnósticos, a maioria dos autores recomendou a indicação ou utilização da timpanometria pelo clínico como método objetivo adjunto, não substitutivo da otoscopia, na decisão da presença ou ausência da efusão na orelha média.1,3,9-12,30-37 Nas crianças cooperativas, a minitimpanometria realizada pelo clínico pode melhorar a acurácia do diagnóstico nos estudos clínicos e na atenção primária, quando não houver certeza do diagnóstico otoscópico.27,33,34 No entanto, assim como ocorre com a OP, a timpanometria raramente é indicada e utilizada pelo clínico na atenção primária à saúde, com o diagnóstico da OMD dependendo principalmente da história clínica, oligo ou assintomática e da otoscopia simples. $1,5,6,9,40-46$

A timpanometria ou reflexometria acústica deve ser indicada pelo clínico para confirmar ou afastar a presença da efusão na orelha média, sempre que não houver certeza do diagnóstico após a realização da otoscopia pneumática, não substituindo, mas complementando de forma objetiva a mesma, no que se refere ao estado da orelha média, aumentando assim a acurácia do diagnóstico.23-25,27,30-39 A timpanometria utilizada pelo clínico diminuiu 30\% dos diagnósticos de OMA, quando utilizada conjuntamente com a otoscopia.5,6,9,45 
Os aspectos relacionados com a habilidade do médico para o diagnóstico da orelha média, através da execução da otoscopia simples e pneumática foram críticos. $9,18,19,40-46$

Parece haver uma enorme diversidade, em vez de concordâncias, entre os profissionais no diagnóstico da otite média, o que dificulta a comparação entre os numerosos estudos nessa área, assim como a discussão sobre a diferenciação entre os quadros, a eficácia do tratamento e as consequências tardias desses quadros. 7,9,40-46

O exame de otoscopia, apesar de método simples e corriqueiro, amplamente utilizado no cotidiano do médico, é permeado por dificuldades, mesmo para especialistas. 5,6,9,40-47 Somente o prejuízo da mobilidade da membrana timpânica foi considerado específico no diagnóstico da efusão da orelha média.22-26 As circunstâncias e os equi pamentos para o diagnóstico, muitas vezes comprometidos, a ansiedade parental, a não cooperação infantil, as dificuldades anatômicas do exame da criança pequena, a ausência de critérios diagnósticos podem interferir negativamente no diagnóstico da otite média com derrame.18,19,29,40-46

A variação diagnóstica entre os diferentes especialistas como o pediatra, médico do programa de saúde da família e o otorrinolaringologista utilizando a otoscopia simples e pneumática reafirmaram a necessidade de formalizar e ampliar o treinamento específico para melhorar a acurácia diagnóstica da OMD.40-46 Assim como é realizado para outros procedimentos médicos, como a coleta de liquor, venoclise, a realização da otoscopia simples e pneumática exige treinamento específico no sentido de aprimorar as competências diagnósticas, com a avaliação rigorosa da proficiência nos procedimentos e diagnóstico das doenças da orelha média incluindo a OMD.9,18,40-46

\section{Conclusões}

A apresentação de questões relevantes para o diagnóstico da OMD permitiu a seleção de problemas

\section{Referências}

1. Rosenfeld R, Culpepper L, Doyle K, Grundfast K, Hoberman A, Kenna M, Lieberthal AS, Mahoney M, Wahl RA, Woods Jr CR, Yawn B. Clinical practice guideline: otitis media with effusion. Otolaryngology - Head and Neck Surgery, 2004;130: S95-S118

2. Paradise J, Rockette D, Colborn K, Bernard B, Smith C, Kurs-Lasky M, Janosky J. Otitis media in 2253 PittsburghArea Infants: prevalence and risk factors during the first importantes, que apontados e discutidos possibilitam a identificação de nós críticos, de relevância para o médico clínico, cuja discussão pode reformular as práticas de saúde relacionadas ao diagnóstico da doença da orelha média.

Os nós críticos importantes na prática clínica se referiram à disparidade das definições do quadro, inconsistência do quadro clínico, falta de padronização dos critérios diagnósticos, questionamento sobre a qualidade do diagnóstico e da proficiência do examinador, que somados às dificuldades peculiares do exame da criança podem contribuir para o diagnóstico inadequado da OMD.

O quadro clínico foi, geralmente, assintomático. Sinais e sintomas, quando presentes, foram subjetivos e atribuídos à perda auditiva. Crianças que frequentam creche, com irmãos mais velhos, pais fumantes, e distúrbios do comportamento ou da audição foram consideradas de risco para a OMD.

Na prática clínica, a detecção da efusão na orelha média (EOM) na ausência de sintomas agudos, pela otoscopia simples (OS) e/ou otoscopia pneumática (OP) realizada em todas as crianças saudáveis e doentes, diagnosticou a OMD. A mobilidade alterada e o aspecto do tímpano pela OP, realizada por médicos qualificados, se mostrou como a ferramenta diagnóstica mais sensível e específica para diagnosticar a OMD. As evidências da presença de efusão foram qualquer um dos itens descritos: a) Pelo menos duas anormalidades do tímpano relacionadas à coloração, opacificação e mobilidade; e/ou b) MT tipicamente retraída, côncava, e com alteração da coloração; e/ou c) Mobilidade reduzida ou ausente pela OP. A distinção entre derrames transitórios (seroso ou purulento) e aqueles persistentes (mucóide) pode orientar o diagnóstico. A timpanometria como método adjunto ou complementar pode aumentar a acurácia diagnóstica da efusão na orelha média, sendo indicada pelo clínico sempre que persistir dúvida no diagnóstico após a realização da otoscopia. two years of life. Pediatrics. 1997; 99: 318-33.

3. Takata G, Chan LS, Mangione-Smtih RM, Agency for Healthcare Research and Quality. Diagnosis, natural history, and late effects of otitis media with effusion. Rockville, MD: Department t. of Health and Human Services, Public Health Service, Agency for Healthcare Research and Quality; 2003. (AHRQ Publication n. 03E023). 
4. Casselbrant ML, Mandel EM. Epidemiology. In: Rosenfeld RM, Bluestone CD, editors. Evidence-based otitis media. 2. ed. Hamilton, Ontario: BC Decke; 2003. p. 147-62.

5. Gonzalez-Vallejo C, Sorum PC, Stewart TR, Chessare JB, Mumpower JL. Physicians'diagnostic judgments and treatment decisions for acute otitis media in children. Med Decis Mak. 1998; 18: 149-62.

6. Fischer TF, Singer AJ, Gulla J, Garra G, Rosenfeld R Reaction toward a new treatment paradigm for acute otitis media. Pediatr Emerg Care. 2005; 21: 170-2.

7. Berman S. Management of otitis media outcomes related to language, behavior, and attention: is it time to change our approach? Pediatrics. 2001; 107: 1175-7.

8. Alho OP, Oja H, Koivu M, Sorri M. Chronic otitis media with effusion in infancy: how frequent is it? How does it develop? Arch Otolaryngol Head Neck Surg. 1995; 121: 432-6.

9. Blomgren K, Pitkäranta A. Is it possible to diagnose acute otitis media accurately in primary health care? Fam Pract. 2003; 20: 524-7.

10. SIGN (Scottish Intercollegiate Guidelines Network). Diagnosis and management of childhood otitis media in primary care. A national clinical guideline. Edinburgh, Scotland: 2003. (SIGN Publication, n. 66)

11. CCHMC (Cincinnati Children's Hospital Medical Center). Evidence based clinical practice guideline for medical management of otitis media with effusion in children 2 months to 13 years of age. Cincinnati, Ohio; 2004

12. UMHS (University of Michigan Health System). Otitis media. Ann Arbor, MI: 2007.

13. Caffarelli C, Savini E, Giordano S, Gianlupi G, Cavagni G. Atopy in children with otitis media with effusion. Clin Exp Allergy. 1998; 28: 591-6.

14. Golz A, Netzer A, Angel-Yeger B, Westerman ST, Gilbert LM, Joachims HZ. Effects of middle ear effusion on the vestibular system in children. Arch Otolaryngol Head Neck Surg. 1998; 119: 695-9.

15. Casselbrandt ML, Furman JM, Mandel EM, Fall PA, KursLasky M, Rockette HE. Past history of otitis media and balance in four-year-old children. Laryngoscope. 2000; 110: $773-8$

16. Roberts JE, Rosenfeld RM, Zeisel SA. Otitis media and speech and language: a meta-analysis of prospective studies. Pediatrics. 2004; 113 (3 Pt 1): e238-48.

17. Rosenfeld RM, Goldsmith AJ, Madell JR. How accurate is parent rating of hearing for children with otitis media. Arch Otolaryngol Head Neck Surg. 1998; 124: 989-92.

18. Pelton S. Otoscopy for the diagnosis of otitis media. Pediatr Infect Dis J. 1998; 17: 540-3.

19. Endo LH, Curi SB. Otoscopia e timpanometria no diagnóstico de otite média secretora. J Pediatr. 1998; 74: 353-4.

20. Franche GL, Tabajara LM, Arrarte JL, Saffer M. Otoscopy and tympanometry in the diagnosis of secretory otitis media. J Pediatr. 1998; 74: 365-7.

21. Engel J, Antenius L, Chenault M, Marres E. Otoscopic findings in relation to tympanometry during infancy. Eur Arch Otorhinolaryngol. 2000; 257: 366-71.

22. Melker RA. Evaluation of the diagnostic value of pneu- matic otoscopy in primary care using the results of tympanometry as a reference standard. Br J Gen Pract. 1993; 43: $22-4$.

23. Alho PO, Koivunen P, Luotonen J. Diagnostic criteria for otitis media in children. Oto-Rhino-Laryngol Nova. 1998; 8:123-9.

24. Guo YC, Shiao AS. Diagnostic methods for otitis media with effusion in children. Zhonghua Yi Xue Za Zhi. 2002; 65: 372-7.

25. Nozza RJ, Bluestone CD, Kardatzke D, Bachman R Identification of middle ear effusion by aural acoustic admittance and otoscopy. Ear Hear. 1994; 15: 310-23.

26. Koivunen P. Detection of effusion and influence on hearing. 1999. [consulted on: 2007 april 6]. Available from: http://herkules.oulu.fi/isbn9514252314/

27. Field M, Allison R, Corwin P, White PS, Doherty J. Microtympanometry, microscopy and tympanometry in evaluating middle ear effusion prior to myringotomy. $\mathrm{N} \mathrm{Z}$ Med J. 1993; 106: 386-7.

28. Isaac ML, Oliveira JAA, Holanda F. Importance of otomicroscopy and acoustic imittance measurements for the early detection of effusion in the middle ear of asymptomatic children in a pediatric outpatient clinic. Rev Bras Otorrinilaringol.1999; 65: 122-7.

29. Barriga F, Schwartz RH, Hayden GF. Adequate illumination for otoscopy. Variations due to power source, bulb, and head and speculum design. Am J Dis Child. 1986; 140: 1237-40.

30. Sassen ML, Van Aaren A, Grotte JJ. Validity of tympanometry in diagnosis of middle ear effusion. Clin Otolaryngol. 1994; 19: 185-9.

31. Palmu A, Puhakka H, Rahko T, Takala AK. Diagnostic value of tympanometry in infants in clinical practice. Int $\mathrm{J}$ Pediatr Otorhinolaryngol. 1999; 49: 207-13.

32. Watters GWR, Jones JE, Freeland A.P. The predictive value of tympanometry in the diagnosis of middle ear effusion. Clin Otolaryngol Allied Sci. 1997; 22: 343-5.

33. Koivunen P, Alho OP, Uhari M, Niemela M, Luotonen J. Minitympanometry indetection middle ear fluid. J Pediatr. 1997; 131: 419-22.

34. van Balen FA, De Melker RA. Validation of a portable tympanometer for use in primary care. Int J Pediatr Otorhinolaryngol. 1994; 29: 219-25.

35. Palmu A, Puhakka H, Rahko T, Takala A, Kilpi T. Predicting the development and outcome of otitis media by tympanometry. Int J Pediatr Otorhinolaryngol. 2002; 62: 135-42.

36. Palmu A, Puhakka H, Huhtala H, Takala AK, Kilpi T. Normative values for tympanometry in 7-and 24-month-old children. Audiology. 2001; 40: 178-84.

37. MrC. Multi-Centre Otitis Media Study Group. Sensitivity, specificity and predictive value of tympanometry in predicting a hearing impairment in otitis media with effusion. Clin Otolaryngol Allied Sci. 1999; 24: 294-300.

38. Block SL, Mandel E, McLinn S, Pichichero ME, Bernstein S, Kimball S, Kozikowski. Spectral gradient acoustic reflectometry for the detection of middle ear effusion by pediatricians and parents. Pediatr Infect Dis J. 1998; 17: 560-4. 
39. Silva CF, Salas RN, Friant M, Silva J. Reflectometría acústica: un nuevo método para estudio de efusión en el oído medio en pediatría. Rev Chil Pediatr. 2002; 73: 369-74

40. Silva AB, Hotaling AJ. A protocol for otolaryngology-head and neck resident training in pneumatic otoscopy. Int $\mathrm{J}$ Pediatr Otorhinolaryngol. 1997; 40: 125-31.

41. Jones WS, Kaleida PH. How helpful is pneumatic otoscopy in improving diagnostic accuracy? Pediatrics. 2003; 112 510-3.

42. Pichichero ME, Poole MD. Assessing diagnostic accuracy and tympanocentesis skills in the management of otitis media. Arch Pediatr Adolesc Med. 2001; 155: 1137-42.

43. Pichichero ME. Diagnostic accuracy, tympanocentesis training performance, and antibiotic selection by pediatric residents in management of otitis media. Pediatrics. 2002; 110: 1064-70.
44. Pichichero M. Diagnostic accuracy of otitis media and tympanocentesis skills assessment among pediatricians. Eur J Clin Microb Infect Dis. 2003; 22: 519-24.

45. Steinbach WJ, Sectish TC. Pediatric resident training in the diagnosis and treatment of acute otitis media. Pediatrics. 2002; 109: 404-8

46. MacClements JE, Parchman M, Passmore C. Otitis media in children: use of diagnostic tools by family practice residents. Fam Med. 2002; 34: 598-603.

47. Eavey RD, Santos JI, Arriaga MA, Gliklich R, Odio C, Desmond MS, Villasenor A, Beltran S, Orloff L, Stool SE. An education model for otitis media care field-tested in Latin America.Otolaryngol Head Neck Surg. 1993; 109: 859-8.

48. Post JC. Direct evidence of bacterial biofilms in otitis media. Laryngoscope. 2001; 111: 2083-94.

Recebido em 30 de abril de 2009

Versão final apresentada em 4 de janeiro de 2010

Aprovado em 22 de fevereiro de 2010 